\title{
ARTICLE Selective expression of constitutively activated STAT6 in intestinal epithelial cells promotes differentiation of secretory cells and protection against helminths
}

\author{
Christoph Schubart ${ }^{1}$, Branislav Krljanac ${ }^{1}$, Manuel Otte ${ }^{1}$, Cornelia Symowski ${ }^{1}$, Eva Martini ${ }^{2}$, Claudia Günther $^{2}$, Christoph Becker $^{2}$, \\ Christoph Daniel ${ }^{3}$ and David Voehringer ${ }^{1}$
}

Intestinal epithelial cells (IECs) constitute an important barrier between host and pathogen. Immune mechanisms that provide protection against gastrointestinal helminths often require IL-4Ra-induced activation of STAT6-regulated genes in IECs. However, it is not known whether STAT6 activation in IECS enhances protective immunity against helminths. Furthermore, the regulation of proliferation and differentiation processes of the intestinal epithelium by IEC-intrinsic STAT6 signaling remains unclear. To address these questions, we generated mice with specific expression of a constitutively active version of STAT6 in IECs. These VillinCre_STAT6vt mice show accumulation of secretory IECS, increased proliferation of IECs and lengthening of the small intestine. They rapidly expelled Nippostrongylus brasiliensis worms even in the absence of T cells. Furthermore, primary infection with Heligmosomoides polygyrus resulted in larval trapping in the submucosa and the fecundity of adult worms was severely impaired. Our results reveal an important IEC-intrinsic role of STAT6-regulated genes for intestinal homeostasis and protective immunity against helminths.

Mucosal Immunology (2019) 12:413-424; https://doi.org/10.1038/s41385-018-0107-3

\section{INTRODUCTION}

Infections with gastrointestinal helminths affect $>3$ billion people worldwide, mainly in tropical countries with low hygiene standards and poor sanitary infrastructure. 'The human hookworm species Necator americanus accounts for $85 \%$ of all human hookworm infections and affects about 650 million people causing 65,000 deaths per year. ${ }^{2}$ The infection cycle, morphology, and transcriptome of $N$. americanus is very similar to Nippostrongylus brasiliensis $(\mathrm{Nb})$, a "rodent hookworm" naturally occurring in rats and mice. ${ }^{3}$ Free-living third-stage larvae (L3) penetrate the skin, migrate to the lung, get coughed up and swallowed, and mature into adult worms in the lumen of the small intestine (SI) by day 4-5 after infection. Adult worms are expelled after about 10 days that is normally dependent on CD4 T cells. ${ }^{4}$ In addition, interleukin (IL)-4 or IL-13 production from cells of the innate immune system such as group 2 innate lymphoid cells (ILC2s) is critical for worm expulsion during primary infection. ${ }^{5-7}$ It appears that tuft cells, a subset of intestinal epithelial cells (IECs), sense the presence of helminths and produce IL-25. This causes accumulation of ILC2s and probably other IL-4-/IL-13-secreting cell types in the lamina propria (LP) to promote goblet cell hyperplasia and tuft cell differentiation. ${ }^{8-10}$

In contrast to this transient infection model, oral infection with L3 of Heligmosomoides polygyrus bakeri $(\mathrm{Hp})$ results in chronic infection. Here the L3 first migrate into the submucosa of the SI from where they return to the intestinal lumen by day 8-9 after infection. ${ }^{11}$ During secondary infection, L3 are trapped in granulomas in the submucosa preventing the maturation of adult worms. ${ }^{12}$ This protective immune mechanism requires memory $\mathrm{T}$ helper type 2 (Th2) cells and Arginase 1-expressing alternatively activated (M2) macrophages. ${ }^{13}$ In addition, antibodies of the immunoglobulin $\mathrm{G}(\mathrm{lgG})$ and $\lg A$ class have been shown to prevent adult worm development. ${ }^{14}$ We could previously demonstrate that IgE-activated release of IL-4/IL-13 from basophils is another important factor for protective immunity during secondary infection with $\mathrm{Hp}$ or $\mathrm{Nb} .^{15}$

The IL-4Ra chain is part of the receptors for IL-4 and IL-13 and signals mainly by Janus kinase (Jak)-mediated activation of STAT6. ${ }^{16}$ Using conditional IL-4Ra knockout mice, it was shown that IL-4/IL-13 signaling in IECs plays a critical role for protective immunity in both helminth infection models. ${ }^{17}$ Other IL-4/IL-13regulated mechanisms such as intestinal peristalsis are also important because deletion of IL-4Ra on smooth muscle cells resulted in delayed worm expulsion in the $\mathrm{Nb}$ model. ${ }^{18}$

At present, it remains unclear whether activation of the STAT6 signaling pathway specifically in IECs fosters accumulation of secretory IECs and protective immunity against gastrointestinal helminths. Therefore, we generated mice in which a constitutively active version of signal transducer and activator of transcription factor 6 (STAT6) is only expressed in IECs. The SI of VillinCre_STAT6vt mice showed goblet cell hyperplasia, accumulation of tuft cells and Paneth cells, higher spontaneous turnover of IECs, and

\footnotetext{
${ }^{1}$ Department of Infection Biology, University Hospital Erlangen and Friedrich-Alexander University Erlangen-Nuremberg (FAU), Erlangen, Germany; ${ }^{2}$ Department of Medicine 1, University Hospital Erlangen and Friedrich-Alexander University Erlangen-Nuremberg (FAU), Erlangen, Germany and ${ }^{3}$ Nephropathology, University Hospital Erlangen and Friedrich-Alexander University Erlangen-Nuremberg (FAU), Erlangen, Germany Correspondence: David Voehringer (David.Voehringer@uk-erlangen.de)
}

Received: 4 June 2018 Accepted: 30 October 2018

Published online: 16 November 2018 
414

increased length. $\mathrm{Nb}$ infection resulted in faster worm expulsion even in the absence of CD4 T cells. Hp infection of VillinCre_STAT6vt mice revealed trapping of L4 stage larvae in the submucosa associated with high expression of Arginase 1 and other markers of M2 macrophages. In addition, we observed reduced fecundity of adult worms in the lumen of the SI.

These results demonstrate that selective activation of STAT6 in IECs provides protective immunity to helminths by larval trapping in the submucosa and affecting fecundity of adult worms in the intestinal lumen.

\section{RESULTS}

Expression of constitutively active STAT6 in IECs of VillinCre_STAT6vt mice

To investigate the effects of STAT6-regulated gene expression in IECs for intestinal homeostasis and protective immunity against gastrointestinal helminths, we first generated mice that express a FLAG-tagged and constitutively active form of STAT6 (STAT6vt) in the Rosa26 locus behind a loxP-flanked Stop cassette (Supplemental Fig. 1). STAT6vt contains two amino acid changes in the $\mathrm{SH} 2$ domain (V547A/T548A) resulting in Jak1/Jak3-independent phosphorylation of Tyr-641, which is critical for dimerization, nuclear translocation, and DNA binding. ${ }^{19,20}$ These Rosa26 $6^{\mathrm{LSL}-}$ STAT6vt mice were crossed to VillinCre mice to generate VillinCre_STAT6vt mice for selective expression of constitutively active STAT6 in IECs.

STAT6vt was expressed in the SI of VillinCre_STAT6vt mice but not in STAT6vt control mice as demonstrated by anti-FLAG western blot (Fig. 1a). Western blots for total STAT6 and phosphorylated STAT6 (pSTAT6) further revealed that the total STAT6 protein level was comparable between VillinCre_STAT6vt and control mice while pSTAT6 was only observed in the SI of VillinCre_STAT6vt mice (Fig. 1b). We further isolated IECs from the SIs and performed immunofluorescence stainings for pSTAT6 and EpCAM as epithelial cell marker to investigate the distribution of pSTAT6 inside IECs. The highest staining intensity for PSTAT6 was found at the apical and basolateral areas of the cells with a weaker staining in the cytoplasm and nucleus of VillinCre_STAT6vt mice while pSTAT6 was basically undetectable in IECs from STAT6vt mice (Fig. 1c).

These results demonstrate that pSTAT6 is constitutively and selectively expressed in IECs of VillinCre_STAT6vt mice.

Expansion of secretory IECs in the SI of VillinCre_STAT6vt mice Since IL-4/IL-13-mediated STAT6 signaling is known to promote goblet cell hyperplasia and accumulation of tuft cells in the SI during helminth infection, we next investigated whether the expression of a constitutively active form of STAT6 in IECs of naive VillinCre STAT6vt mice promotes differentiation and turnover of secretory IECs. Therefore, we performed histological analysis and reverse transcriptase-polymerase chain reaction (RT-PCR) experiments with samples from the duodenum, jejunum, and ileum and characteristic markers for goblet cells, tuft cells, and Paneth cells. VillinCre_STAT6vt mice contained more goblet cells, more tuft cells, and more Paneth cells as compared to STAT6vt mice, especially in the jejunum and ileum (Fig. 2a). Goblet cells and Paneth cells appeared hyperplastic indicating their active production of effector molecules (Fig. 2a and Supplemental Fig. 2). This difference was also reflected by increased mRNA expression levels of Relm- $\beta$ (goblet cell marker), IL-25 (tuft cell marker), and Angiogenin-4 (Ang4; Paneth cell marker) (Fig. 2b). The higher expression of Relm- $\beta$ in VillinCre_STAT6vt mice was further confirmed at the protein level with western blots of fecal samples (Fig. 2c).

Increased numbers of tuft cells do not result in accumulation of ILC2s in the LP of VillinCre_STAT6vt mice

Tuft cells are the major source of IL-25 in the SI and studies with helminth infection models indicated that tuft cell-derived IL-25 promotes accumulation of ILC2s in the LP. ${ }^{8-10}$ Therefore, we expected to find more ILC2s in the LP of VillinCre_STAT6vt mice as compared to STAT6vt control mice. However, histological analysis revealed that unlike the situation in helminth-infected wild-type mice ILC2s were not increased in the LP of naive VillinCre_STAT6vt mice despite massive expansion of tuft cells (Fig. 3a). Furthermore, we performed flow cytometric analysis to determine the frequency of ILC2s in both mouse strains. No obvious increase of ILC2s in VillinCre_STAT6vt mice was found by this approach either, indicating that (1) the increased number of tuft cells in the LP of VillinCre STAT6vt mice is not dependent on recruitment of ILC2s and (2) more tuft cells are not sufficient to promote ILC2 accumulation in the LP (Fig. $3 \mathrm{~b}$ and Supplemental Fig. 3a). Flow cytometric analysis of other effector cell subsets further revealed reduced frequencies of eosinophils, neutrophils, and $\mathrm{CD} 4^{+} \mathrm{T}$ cells in the LP of VillinCre_STAT6vt as compared to STAT6vt mice, while no difference was observed for basophils (Fig. $3 \mathrm{c}$ and Supplemental Fig. 3a).

IEC-intrinsic PSTAT6 promotes IEC turnover and lengthening of the SI

The increased number of all three subsets of secretory IECs in VillinCre_STAT6vt mice suggested that IEC-intrinsic pSTAT6 promotes proliferation and differentiation of IEC. To directly investigate the turnover of IECs in vivo, we injected bromodeoxyuridine (BrdU) intraperitoneally (i.p.) and analyzed samples from the ileum $24 \mathrm{~h}$ later by immunofluorescence staining. By measuring the distance between the crypts and the most distal BrdUlabeled IECs, we observed that almost twice as many IECs were generated during this time frame in VillinCre_STAT6vt mice as compared to STAT6vt control mice (Fig. 4a). The increased production of IECs also led to an increase of the total length of the SI (Fig. 4b). These changes are unlikely to be caused by differences in the stem cell population as the mRNA level for the stem cell marker Leucin-rich repeat-containing G-protein-coupled receptor 5 (Lgr-5) was comparable (Fig. 4c).

We further investigated whether STAT6 signaling interferes with the Notch signaling pathway, which is known to inhibit differentiation of secretory IECs. ${ }^{21}$ Therefore, we analyzed the expression levels of Delta-like canonical Notch ligand 4 (DII4), the Notch-signaling activator protein-O-fucosyltransferase 1 (Pofut1), the Notch-target gene hes family bHLH transcription factor 1 (Hes1), and the secretory IEC-promoting transcription factors atonal bHLH transcription factor 1 (Math1)/Atoh1 and SAM pointed domain containing ets transcription factor (Spdef). We observed no difference for the expression of DII4, Pofut1, and Hes 1 in VillinCre_STAT6vt mice, while Math1 and Spdef were unexpectedly downregulated in comparison to control mice (Fig. 4c). Therefore, we can conclude that pSTAT6 does neitherinhibit Notch signaling nor promote Math1 or Spdef expression. However, we observed upregulation of SRY (sex determining region Y)-box 4 (Sox-4) and Sox-9, which can promote development of secretory IECs independently of Math1 $1^{22,23}$ (Fig. 4c).

Next, we investigated whether increased proliferation and enhanced differentiation of secretory IECs can also be observed in organoid cultures in vitro. Therefore, we set up organoid cultures from VillinCre_STAT6vt mice and STAT6vt control mice as described in Methods. Cultures were analyzed by light microscopy on days 2 and 7 to determine the size and branching of organoids. We observed no obvious differences between organoids generated from both mouse strains (Fig. 4d). As expected, western blot analysis revealed that STAT6 was constitutively phosphorylated in organoids from VillinCre_STAT6vt mice (Fig. 4e). Expression of the Paneth cell marker Ang4 was higher in organoids from VillinCre_STAT6vt mice while markers for goblet cells and tuft cells were not increased as determined by quantitative RT-PCR (Fig. 4f). This indicates that unknown in vivo factors are required 

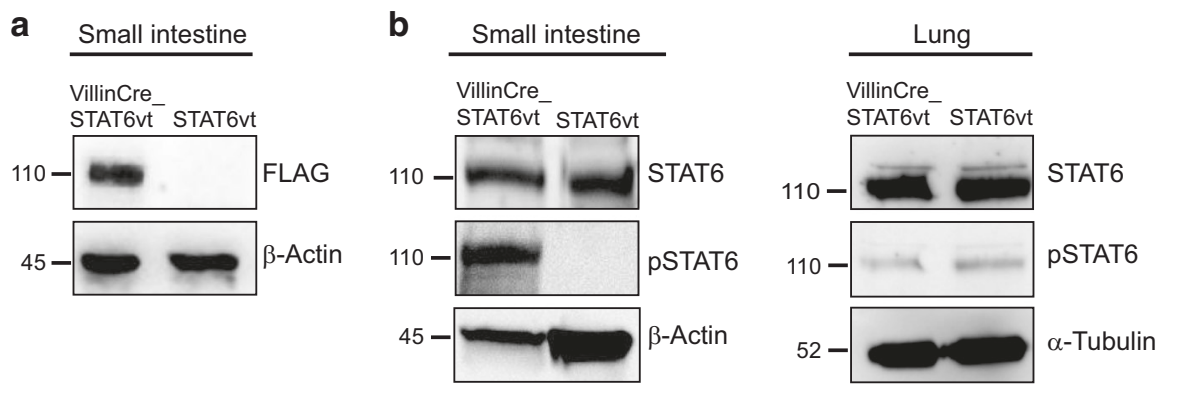

C

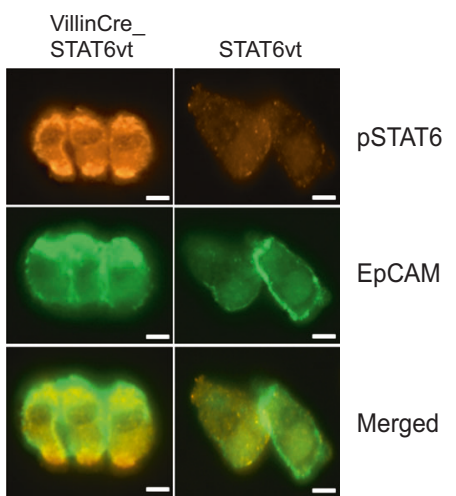

Fig. 1 Expression of STAT6vt protein leads to constitutive phosphorylation of STAT6 in intestinal epithelial cells of VillinCre_STAT6vt mice a, b Immunoblot of the intestine and lungs from naive VillinCre_STAT6vt mice and their control littermates (STAT6vt). Samples were analyzed for Cterminal FLAG tag a, total STAT6 (STAT6-M20, b, upper row), and phosphorylated STAT6 (pSTAT6, b, middle row). $\beta$-Actin and and $\alpha$-Tubulin served as reference proteins. c Isolated intestinal epithelial cells from naive VillinCre_STAT6vt mice (left) and their control littermates (right) were stained for phosphorylated STAT6 (pSTAT6, orange) and the epithelial cell marker EpCAM (green). Blots are representative of one out of two independent experiments with two mice per group per experiment. Numbers indicate the molecular weight in kD. The picture shows one experiment with two mice per group. Scale bars represent $20 \mu \mathrm{m}$

for pSTAT6-mediated differentiation of goblet cells and tuft cells while this is not the case for Paneth cells.

VillinCre_STAT6vt mice efficiently expel $\mathrm{Nb}$ even in the absence of CD4 T cells

The observed changes in the SI are reminiscent of infections with gastrointestinal helminths where goblet cell hyperplasia and expansion of tuft cells is associated with efficient worm expulsion. Therefore, we investigated whether VillinCre_STAT6vt mice show improved protective immunity against helminths as compared to STAT6vt control mice. We infected mice with $\mathrm{Nb}$ and analyzed the number of eggs in the feces and adult worms in the SI. The number of eggs on day 6 after infection was comparable between both mouse strains. However, on days 8 and 10 we observed 30-100 times fewer eggs in VillinCre_STAT6vt mice as compared to STAT6vt controls (Fig. 5a). Analysis of worm counts on day 8 revealed almost complete worm expulsion in VillinCre_STAT6vt mice while STAT6vt controls still contained numerous worms (Fig. 5a).

It is well established that CD4 T cells are required for worm expulsion in the $\mathrm{Nb}$ infection model in wild-type mice. To investigate whether $\mathrm{Nb}$ expulsion in VillinCre_STAT6vt mice is also dependent on CD4 T cells or not, we treated mice with an anti-CD4-depleting antibody or isotype control before and during $\mathrm{Nb}$ infection and determined the number of adult worms in the SI on day 10 after infection. Worms were completely eliminated in isotype-treated control mice of both strains. However, anti-CD4treated mice revealed that VillinCre_STAT6vt mice could still expel the worms while STAT6vt control mice could not (Fig. 5b). Interestingly, flow cytometric analysis revealed slightly less ILC2s and more eosinophils and basophils in the LP of $\mathrm{Nb}$-infected VillinCre_STAT6vt as compared to STAT6vt mice (Fig. 5c and Supplemental Fig. 3a). Quantitative RT-PCR analysis further demonstrated lower expression levels of IL-13, IL-25, mucin 5, subtypes A and C, tracheobronchial/gastric (Muc5ac), and resistinlike beta (Retnlb) in VillinCre_STAT6vt mice (Fig. 5d). These results suggest that rapid worm expulsion results in poor induction of the innate type 2 immune response after $\mathrm{Nb}$ infection in VillinCre_STAT6vt mice.

To further investigate the adaptive type 2 immune response, we measured IL-4 and IL-13 production from restimulated CD4 T cells isolated from mesenteric lymph nodes ( $\mathrm{mLNs}$ ) and serum $\mathrm{lgE}$ levels on day 10 after infection. Flow cytometric analysis and enzyme-linked immunosorbent assay (ELISA) showed comparable production of IL-4 and IL-13 from CD4 T cells in VillinCre_STAT6vt mice and STAT6vt control mice (Fig. 5e, $\mathrm{f}$ and Supplemental Fig. $3 b$ ). In addition, serum IgE levels were not significantly different in both strains of mice (Fig. $5 \mathrm{~g}$ ).

We conclude that activated IECs in VillinCre_STAT6vt mice are sufficient for protective immunity against $\mathrm{Nb}$ in the absence of CD4 T cells.The rapid worm expulsion results in a weaker innate type 2 immune response in the SI while induction of adaptive type 2 immunity in draining $\mathrm{mLNs}$ appears to be normal.

Two levels of protective immunity against $H p$ in VillinCre_STAT6vt mice

In contrast to $\mathrm{Nb}$ which does not cross the epithelial barrier and stays in the lumen of the $\mathrm{SI}, \mathrm{L} 3$ of $\mathrm{Hp}$ first migrate into the submucosa where they develop into the L4 stage and stay for 7 days before they migrate back into the intestinal lumen. To investigate whether the activated IECs in VillinCre_STAT6vt mice prevent L3 entry into the submucosa, we analyzed the SI at different times after oral infection. At day 7 after infection, we observed the same number of larvae in the submucosa of both mouse strains indicating that the activated IECS in VillinCre_STAT6vt mice did not prevent larval entry into the submucosa (Fig. 6a). Quantification of granulomas that still contained larvae at day 8 after infection further supported this finding (Fig. 6b). A previous study has shown that M2 macrophages that express Arginase 1 (Arg1) are associated with larval killing in granulomas after secondary infection. ${ }^{13}$ Therefore, we investigated the expression of Arg1 in granulomas by immunofluorescence staining on day 7 after primary infection. Granulomas in VillinCre_STAT6vt mice revealed strong Arg1 expression, whereas this was not the case in STAT6vt control mice (Fig. 6c). Owing to technical limitations, we did not succeed to obtain reliable costaining for Arg1 and a macrophage marker like CD68. However, co-staining for Relm- $a$ and CD68 demonstrated that M2 macrophages were present in granulomas of both strains of mice and in close proximity to the trapped larvae (Fig. 4c). Furthermore, we found no difference for eosinophil recruitment (Fig. 4c). Further analysis by RT-PCR revealed higher expression levels of $M 2$ markers like mannose receptor 1 (Mrc1; CD206), chitinase-like protein 3 (Chil3; Ym1), programmed cell death 1 ligand 2 (Pdcd1lg2; PD-L2), and resistin-like alpha (Retnla; Fizz-1) in granulomas from VillinCre_STAT6vt mice as compared to controls (Fig. $6 \mathrm{~d}$ ). In addition, higher expression levels of collagen I and the Relm-a-inducible enzyme lysyl-hydroxylase 2 (LH2), which 
a

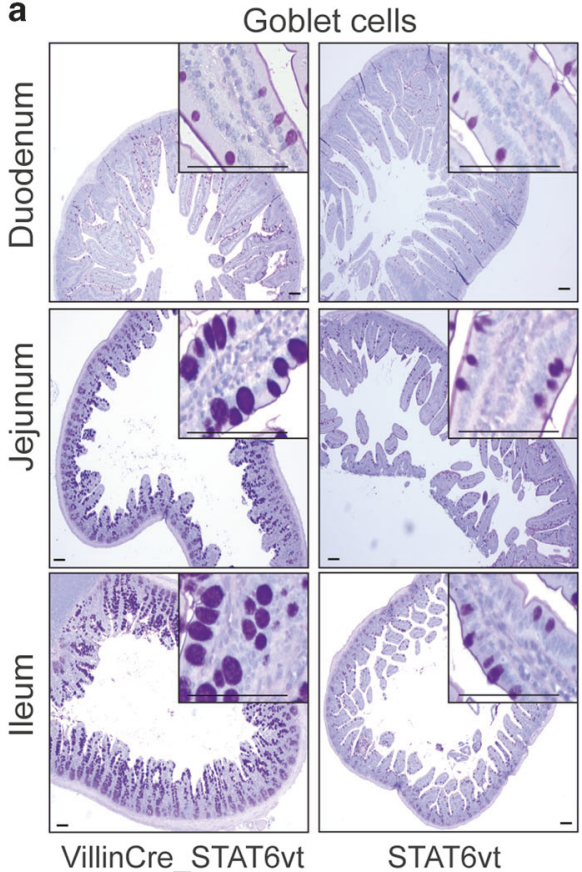

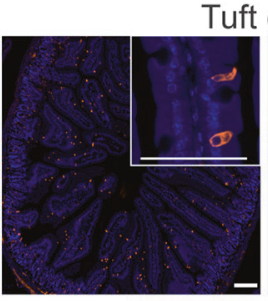
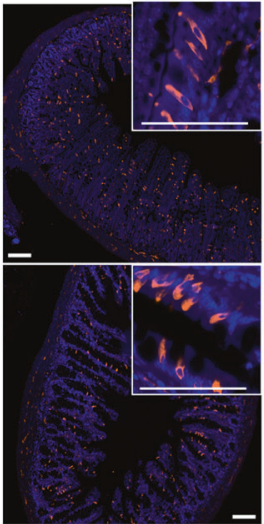

VillinCre_STAT6vt
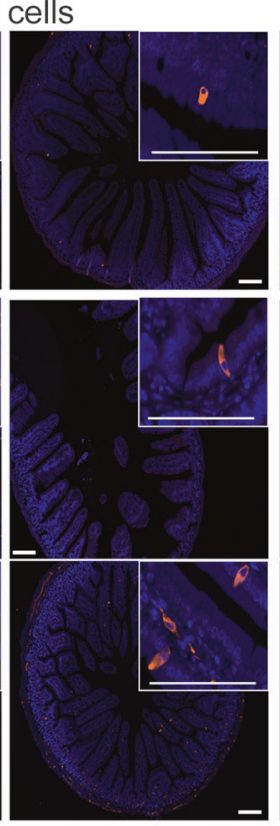

STAT6vt
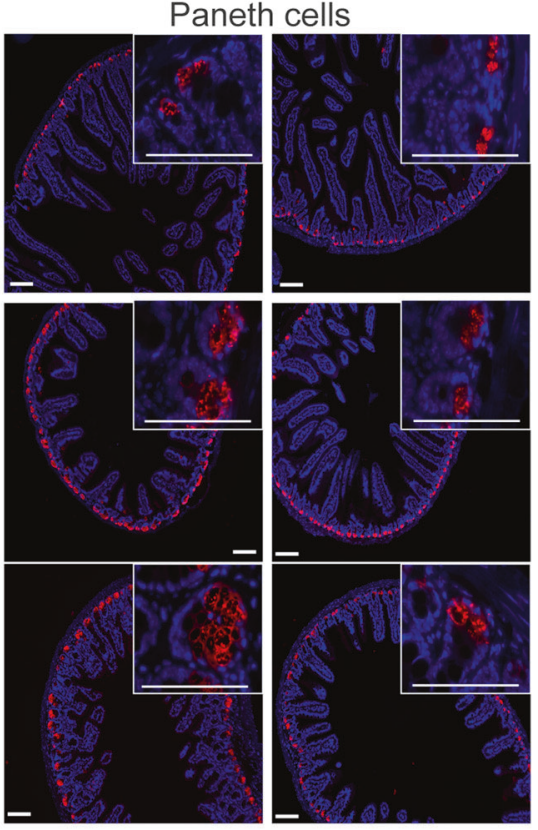

VillinCre_STAT6vt

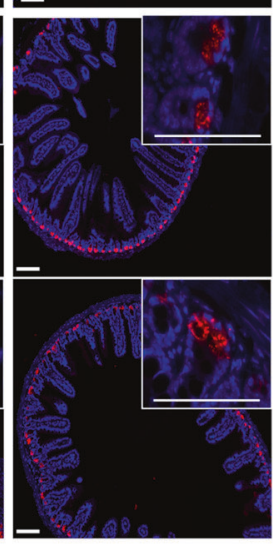

STAT6vt b

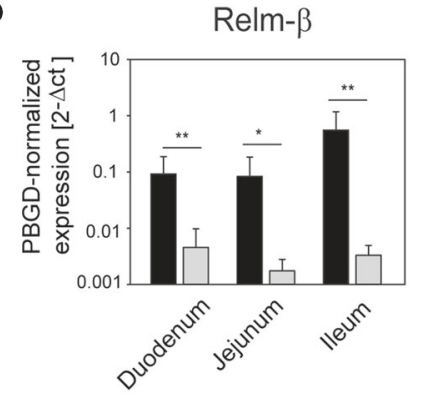

C

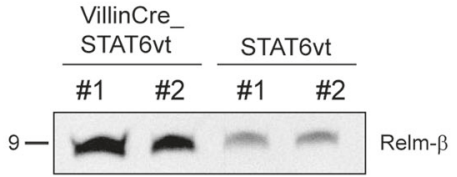

IL-25

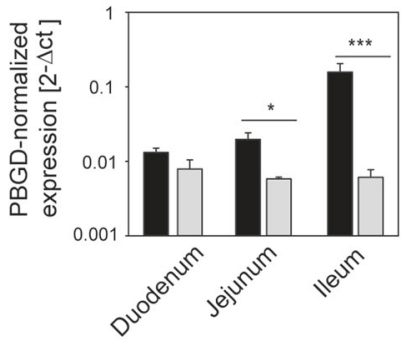

Ang4

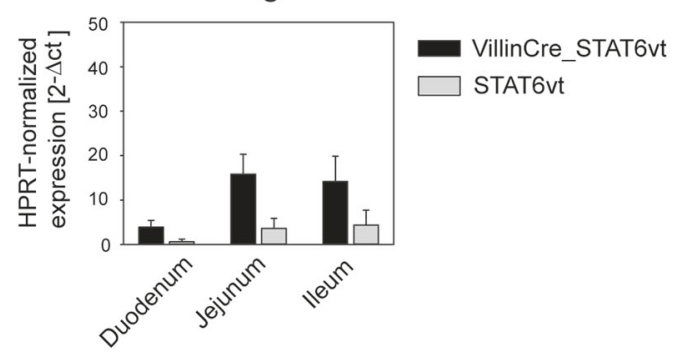

Fig. 2 Increased numbers of secretory intestinal epithelial cells in VillinCre_STAT6vt mice. a Paraffin sections from the duodenum, jejunum, and ileum of naive VillinCre_STAT6vt mice and their control littermates were stained for secretory intestinal epithelial cells. From left to right: PAS and H\&E staining for goblet cells (purple), anti doublecortin-like kinase 1 (DCLK1) staining for tuft cells (orange), and anti-Lysozyme staining for Paneth cells (red). For tuft and Paneth cells, the nuclei were counter stained with DAPI (blue). Magnified views of the cells of interest are in the upper right corner of each picture. Scale bar represents $50 \mu \mathrm{m}$. b mRNA expression of genes associated with secretory intestinal epithelial cells. From left to right: Retnlb (Relm- $\beta$, goblet cells), interleukin-25 (IL-25, tuft cells), angiogenin-4 (Ang4, Paneth cells). PBGD and HPRT1 served as reference genes for normalization. c Western blot for Relm- $\beta$ protein out of fecal pellets of one experiment, \#1 and \#2 represent individual mice per group. Pictures are representative of one out of at least two independent experiments, whereas the bar graphs show the mean + s.e.m. of pooled data from two independent experiments. Two to three mice per group per experiment were used. ${ }^{*} P \leq 0.05,{ }^{* *} P \leq 0.01,{ }^{* * *} P \leq 0.001$, unpaired two-tailed Student's $t$ test

promotes collagen fiber formation, ${ }^{24}$ were observed in granulomas of VilliniCre_STAT6vt mice (Fig. 6e).

The efficient trapping of larvae in the submucosa resulted in very few adult worms in the lumen on day 9 (Fig. 6f). To further investigate whether the fecundity of the few remaining females was normal or not, we took pictures of female worms on day 15 and determined the number of eggs in the feces on days 10, 15, and 20 after primary infection. Females in STAT6vt mice produce thousands of eggs per gram feces while no eggs could be found in the feces of VillinCre_STAT6vt mice at all time points (Fig. 6f). The few detectable female worms in VillinCre STAT6vt mice contained much fewer eggs as compared to worms in control mice demonstrating the reduced fecundity (Fig. 6g). To further determine the viability of the trapped larvae, we recorded movies and measured ATP and protein levels. The movements of L4 larvae isolated from the granulomas on day 7 appeared normal in both strains of mice (Supplemental Movies). We further observed the same ATP levels for L4 larvae at days 7 and 9 after infection of VillinCre_STAT6vt mice, and these values were comparable to L4 larvae and adult worms in STAT6vt control mice (Fig. 6h). In addition, we analyzed mRNA expression levels of type 2 cytokines and effector proteins in granulomas on day 9 after infection. VillinCre_STAT6vt mice produced more IL-4, IL-5, and IL- 13 but less IL-25 and less Muc5ac as compared to STAT6vt control mice 
a

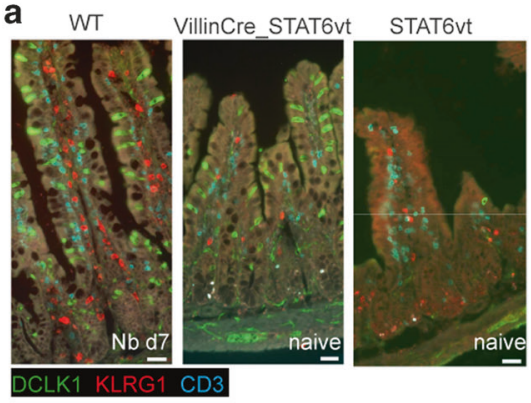

b

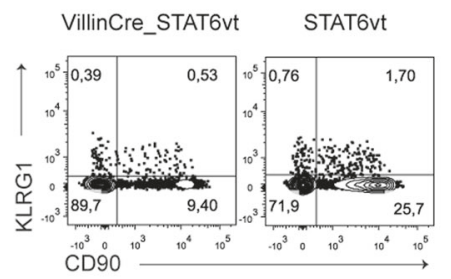

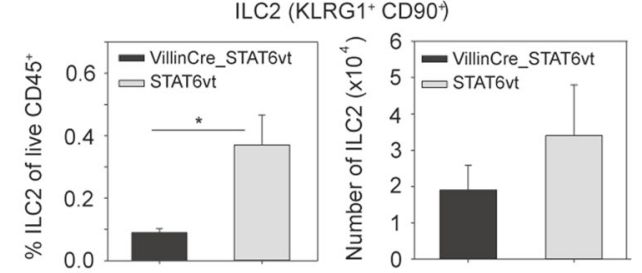

C
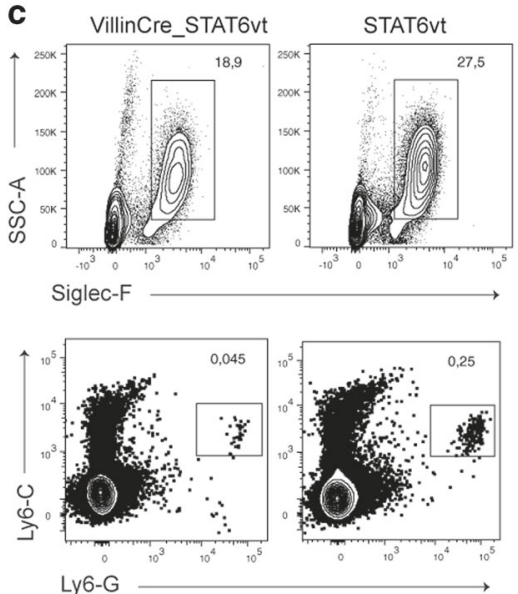

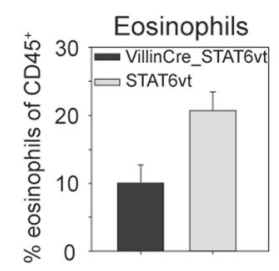

Neutrophils

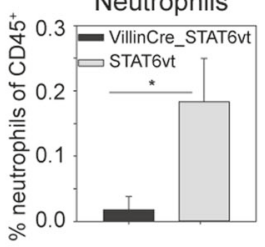

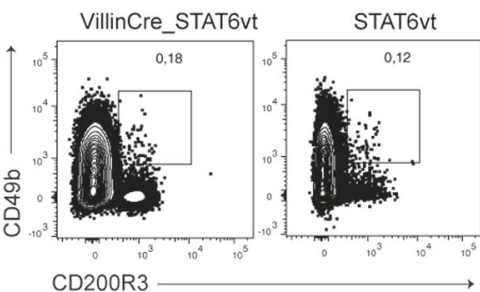

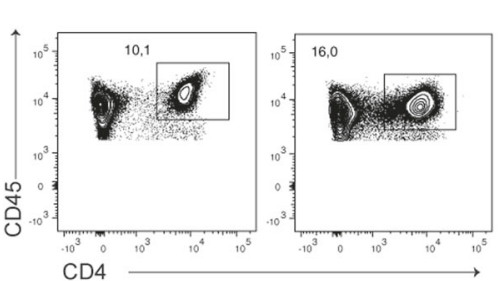

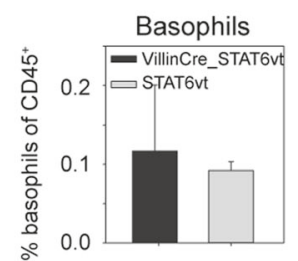

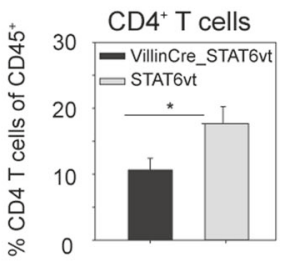

Fig. 3 Tuft cell amplification in mice with constant STAT6 signaling in IECs does not lead to increased influx of ILC2s. a Cryosections from duodenum of wild-type mice on day 7 after primary infection with Nippostrongylus brasiliensis (left) and ileum of naive VillinCre_STAT6vt mice (middle) and their control littermates (right) stained for killer cell lectin-like receptor subfamily G, member 1 (KLRG1, red), DCLK1 (green), and CD3 (turquoise). Scale bars represent $20 \mu \mathrm{m}$. b Representative FACS plots of naive VillinCre_STAT6vt mice and their control littermates analyzed for KLRG $1^{+} \mathrm{CD} 90^{+}$ILC2s gated from $\mathrm{Lin}^{-} \mathrm{CD} 4^{-}$cells in the intestinal lamina propria. $\mathrm{Lin}^{+}$cells include $\mathrm{CD} 3 \mathrm{e}^{+} \mathrm{CD} 8 \mathrm{e}^{+} \mathrm{CD} 11 \mathrm{~b}^{+} \mathrm{CD} 49 \mathrm{~b}^{+}$ $\mathrm{B}_{220^{+}} \mathrm{Gr}^{+} \mathrm{Ter} 19^{+} \mathrm{TCR} \gamma \delta^{+} \mathrm{NK} 1.1^{+}$cells. The bar graphs show the percentage and the total cell count of $\mathrm{KLRG}^{+} \mathrm{CD} 90^{+}$ILC2s of living CD45 ${ }^{+}$ cells. c Representative FACS plots of naive VillinCre_STAT6vt and their control littermates analyzed for SSC-A ${ }^{\text {hi }}$ Siglec- $\mathrm{F}^{+}$eosinophils gated

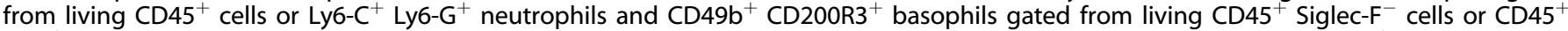
$\mathrm{CD}^{+} \mathrm{T}$ cells gated from living cells. The bar graphs show the percentage of eosinophils, neutrophils, basophils, and CD4 ${ }^{+} \mathrm{T}_{\text {cells }}$ of living $\mathrm{CD} 45^{+}$cells. a, b Representative pictures and plots of one out of two independent experiments and plots of one experiment (c) are depicted. Bar graphs show the mean + s.e.m. of pooled data from two independent experiments $\mathbf{a}, \mathbf{b}$ or the mean + s.d. from one experiment $\mathbf{c}$. Two to three mice per group per experiment were used

(Fig. 6i). Furthermore, we observed a trend toward higher frequencies of macrophages and neutrophils in the LP of day 9infected VillinCre_STAT6vt mice (Fig. 6j). To finally exclude the possibility that impaired fecundity results from the low number of adult worms, we performed infections of wild-type mice with different numbers of L3. An infection with only 20 larvae instead of the usual number of 200 larvae still resulted in hundreds of eggs per gram feces on days 15 and 21 (Fig. 6k).

These results demonstrate that protective immunity against $\mathrm{Hp}$ in VillinCre_STAT6vt mice operates at two levels. First, STAT6activated mechanisms in IECs lead to strong expression of Arg1, several other M2-associated effector molecules, and collagen in larvae-containing granulomas, which prevents their migration back into the intestinal lumen without affecting larval viability. Second, the few worms that escaped this first line of defense and managed to develop into adults in the lumen show severely reduced fecundity. Both mechanisms together provide efficient protection against this parasite.

\section{DISCUSSION}

The intestinal epithelium provides an important barrier function and plays a critical role in protection against infections with various pathogens ranging from viruses to parasitic worms. Using a newly established mouse model, we demonstrate here that expression of constitutively active STAT6 in IECs results in increased proliferation of IECs and accumulation of goblet cells, tuft cells, and Paneth cells in the SI. The modified intestinal environment resulted in improved protective immune responses against gastrointestinal helminths.

STAT6 regulates gene expression downstream of the IL-4Ra chain that together with the IL-13R1 chain constitutes the type II IL-4 receptor that binds IL-4 and IL-13 and is expressed mainly on non-hematopoietic cells. ${ }^{25}$ STAT6-deficient mice show impaired goblet cell hyperplasia and poor worm expulsion after gastrointestinal helminth infections demonstrating a requirement for STAT6-regulated genes in protective type 2 immunity. ${ }^{26-28}$ Furthermore, studies with bone marrow chimeric mice showed that STAT6 expression in non-hematopoietic cells including IECs, smooth muscle cells, and others is important for worm expulsion in the $\mathrm{Nb}$ model. ${ }^{29}$ Selective deletion of IL-4Ra in IECs also resulted in impaired control of primary $\mathrm{Nb}$ infection, indicating that IL-13induced activation of STAT6 in epithelial cells is critical for protective immunity. ${ }^{17}$ However, this finding does not rule out a requirement for STAT6-regulated genes in other cell types. Generating VillinCre_STAT6vt mice with IEC-specific expression of a constitutively active version of STAT6 allowed us to investigate the role of STAT6-regulated genes in IECS for regulation of homeostasis and differentiation of the gut epithelium and how these changes directly affect the control of intestinal helminth infections. 
a VillinCre STAT6vt

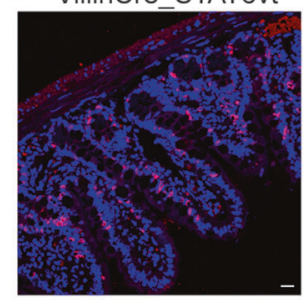

b

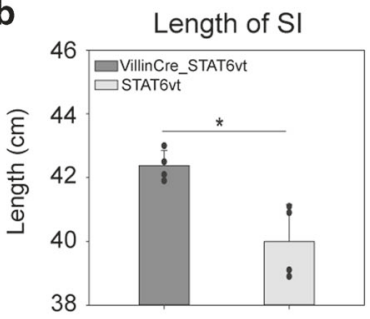

STAT6vt

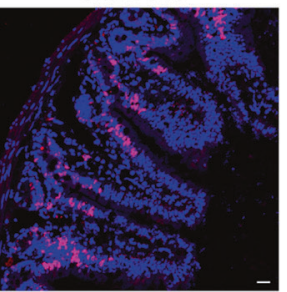

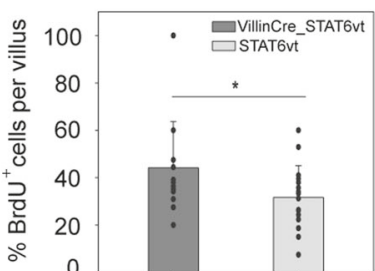

C
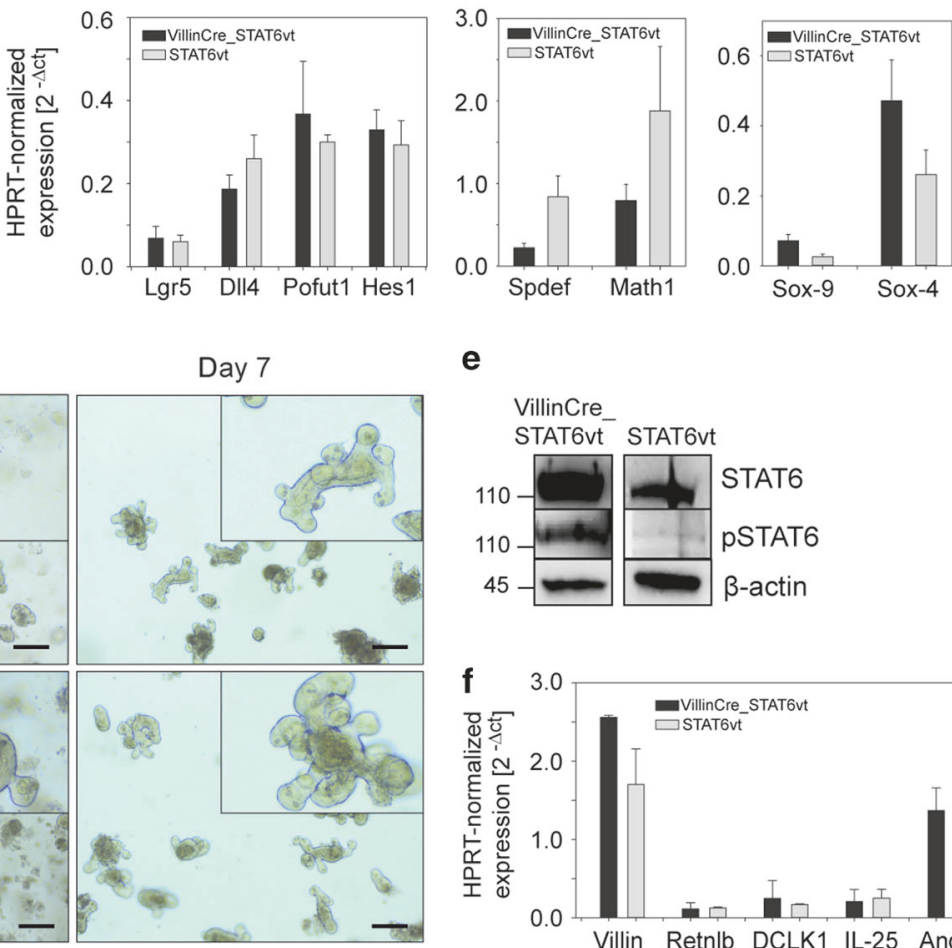

e

Day 7
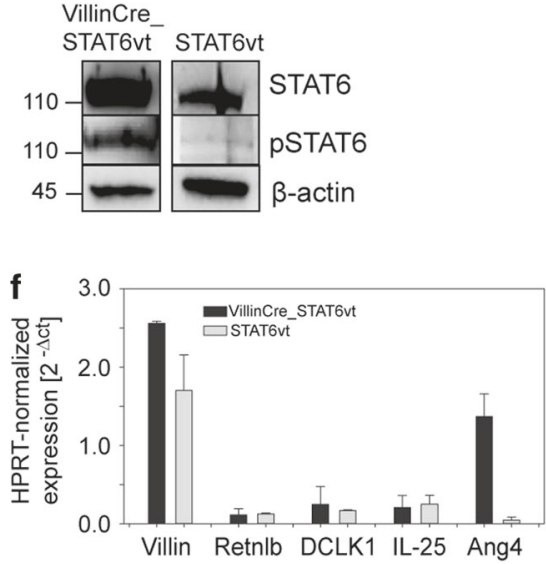

Day 2

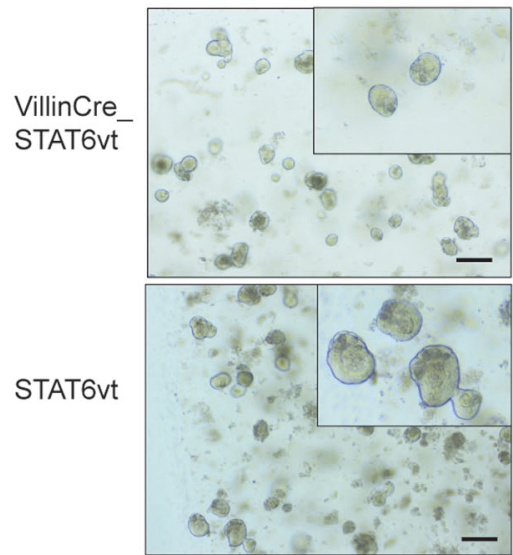

Fig. 4 Activated STAT6 promotes proliferation of IECs in VillinCre_STAT6vt mice but does not affect the number of stem cells. a Naive VillinCre_STAT6vt and their control litter mates were intraperitoneally injected with BrdU. After $24 \mathrm{~h}$, ileal pieces were collected and cryosections were stained for BrdU (red) and nuclei were counterstained with DAPI (blue). Proliferation was determined by measuring the distance from crypts to the most distant $\mathrm{BrdU}^{+}$cell on the villus axis. In addition, the percentage of proliferating cells was analyzed by counting all $\mathrm{BrdU}^{+}$cells per villus. b Length of the whole small intestine of naive VillinCre_STAT6vt mice (dark gray bar) and their control littermates (light gray bar). c mRNA expression of Lgr5, Dll4, Pofut1, Hes1, Spdef, Math1, Sox-9, and Sox-4in the ileum of naive VillinCre_STAT6vt mice (black bar) and their control littermates (gray bar) was analyzed by quantitative RT-PCR. HPRT1 was used as the reference gene for normalization. d Small intestinal organoids were generated for up to 7 days using crypts from naive VillinCre_STAT6vt mice (top) and their control littermates (bottom). Growth and differentiation was documented every day by light microscopy. e Organoids from 12 wells per mouse were pooled and analyzed by western blot for total STAT6 (upper row) and phosphorylated STAT6 (pSTAT6, middle row). $\beta$ Actin served as reference protein. Numbers indicate the molecular weight in kD. f Organoids from 4 wells per mouse were pooled on day 7 of culture, RNA was isolated, and mRNA expression of Villin, Retnlb, DCLK1, IL-25, and Ang4 genes was analyzed by quantitative RT-PCR. HPRT1 served as the reference gene for normalization. Pictures in $\mathbf{a}$, $\mathbf{d}$ are representative of one out of two independent experiments. Representative pictures in $\mathbf{d}$ show organoids on days 2 and 7 of culture. Bar graphs show the mean + s.e.m of pooled data from two independent experiments a-c or show the mean + s.d. of one experiment f. The western blot is representative for two mice per group from one experiment e. Two to three mice per group per experiment were used. Scale bars represent $20 \mu \mathrm{m} .{ }^{*} P \leq 0.05,{ }^{* *} P \leq 0.01,{ }^{* * *} P \leq 0.001$, unpaired two-tailed Student's $t$ test

The total length of the SI of VillinCre_STAT6vt mice was about $10 \%$ larger as compared to control mice and this correlated with increased BrdU incorporation in IECs. These findings are consistent with a previous report on IL-13-regulated IEC proliferation in the large intestine of mice infected with the helminth Trichuris muris. ${ }^{30}$ Interestingly, intestinal organoids from VillinCre_STAT6vt mice did not show obvious differences in growth or budding phenotypes compared to control mice despite a clear pSTAT6 signal in western blot analysis. This indicates that additional in vivo factors derived from microbiota or hematopoietic cells are required for STAT6-induced proliferation of IECs.

Naive VillinCre_STAT6vt mice further showed increased numbers of goblet cells and tuft cells but surprisingly this did not correlate with accumulation of ILC2s in the LP. Tuft cells are the major source of IL-25 in the SI and IL-25 is a potent inducer of ILC2s, which secrete IL-13 to activate tuft cells and goblet cells. ${ }^{8,9}$ One possible explanation for this unexpected finding could be that tuft cells do not release IL-25 protein spontaneously but require an activating signal that would be present during 


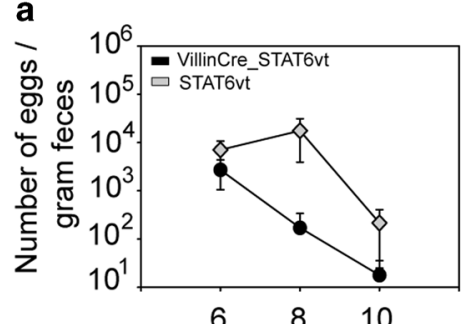

Days after infection

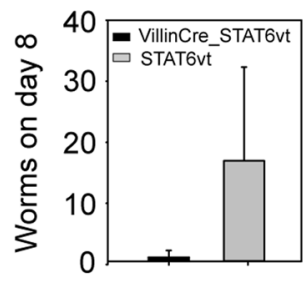

b

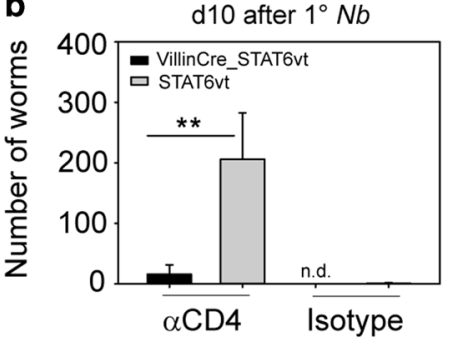

C
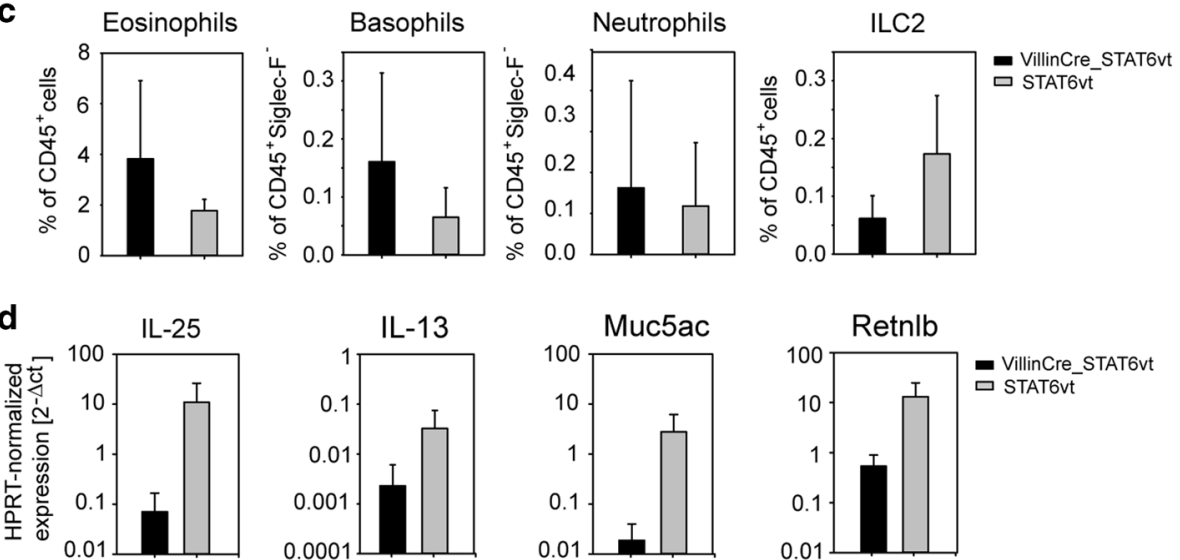

e
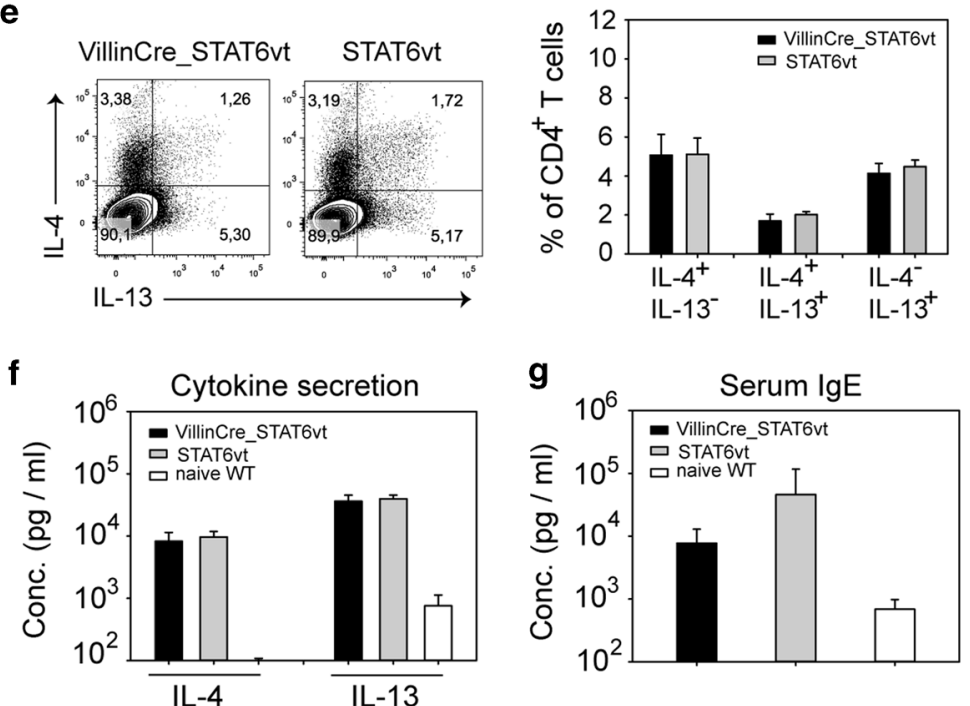

Fig. 5 Faster parasite clearance and normal Th2 response after $\mathrm{Nb}$ infection. VillinCre STAT6vt mice and their control littermates were infected with $\mathrm{Nb}$ L3. a Number of eggs in fecal pellets on days 6,8 , and 10 after infection and number of adult worms in the intestinal lumen on day 8 after infection. b Number of adult worms in the intestinal lumen on day 10 after infection of mice that had been depleted of CD4 T cells ( $\alpha$ CD4) or not (Iso-Ctrl). c Immune cells were isolated out of the LP on day 8 after $\mathrm{Nb}$ infection and analyzed by FACS. The bar graphs show the percentages of Siglec- $\mathrm{F}^{+}$eosinophils from living $\mathrm{CD}^{+} 5^{+}$cells and $\mathrm{CD} 49 \mathrm{~b}^{+} \mathrm{CD} 200 \mathrm{R} 3^{+}$basophils and $\mathrm{Ly}^{+}-\mathrm{C}^{+}$Ly6- $\mathrm{G}^{+}$neutrophils from living $\mathrm{CD}_{4}{ }^{+}$Siglec-F${ }^{-}$cells. $\mathrm{CD} 90^{+} \mathrm{KLRG}^{+} \mathrm{Lin}^{-} \mathrm{ILC2}$ s were gated from living CD45 ${ }^{+} \mathrm{Lin}^{-}$cells. $\mathbf{d}$ mRNA expression of genes associated with a type 2 immune response on day 8 after $N b$ infection. HPRT served as the reference gene for normalization. e Intracellular staining for IL-4 and IL-13 production by $\mathrm{CD}^{+} \mathrm{T}$ cells from mesenteric lymph nodes of day $10 \mathrm{Nb}$-infected mice. Dot plots are gated on single live CD4 ${ }^{+}$cells and gates of the stimulated samples were set according to the respective unstimulated control. Bar graphs show the frequency of IL-4-positive IL-13negative, IL-4/IL-13 double positive, and IL-4-negative IL-13-positive CD4 ${ }^{+}$T cells. $f$ Amounts of IL-4 and IL-13 in the supernatant of anti-CD3/ anti-CD28 restimulated T cells quantified by ELISA. $\mathbf{g}$ IgE levels in the serum of day $10 \mathrm{Nb}$-infected mice quantified by ELISA. a-g Black symbols/bars represent VillinCre STAT6vt mice, gray symbols/bars represent their control littermates, and white columns represent WT C57BL/ 6 mice. $\mathbf{a}, \mathbf{b}, \mathbf{e}-\mathbf{g}$ Bars and symbols show the mean + s.e.m of pooled data from at least two independent experiments. a (worm count day 8), $\mathbf{c}$, d Bars show the mean + s.d. from one experiment. e Dot plots are representative of one out of two independent experiments. Two to four mice per group per experiment were used for the experiments. ${ }^{*} P \leq 0.05,{ }^{* *} P \leq 0.01,{ }^{* * *} P \leq 0.001$, unpaired two-tailed Student's $t$ test 

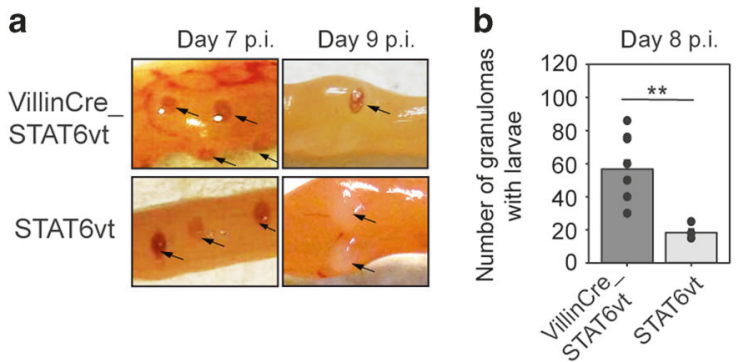

d

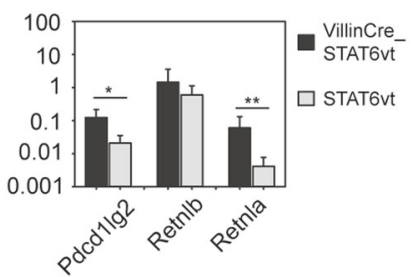

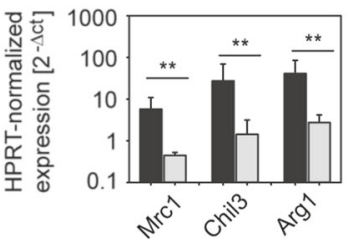

C.

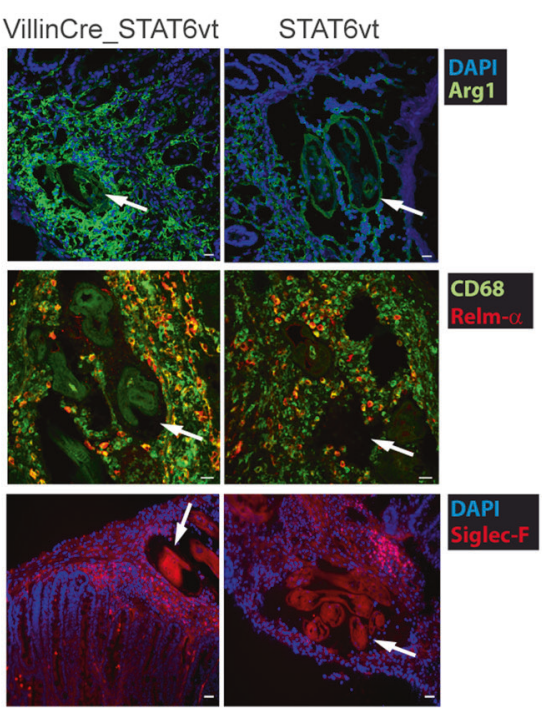

f
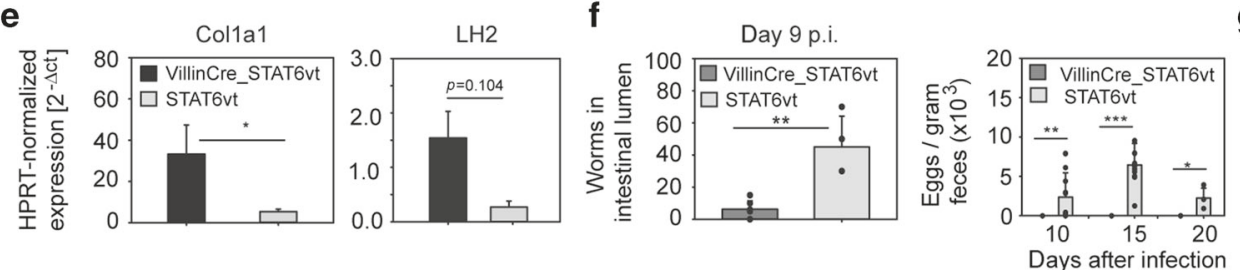

$\mathbf{g}_{V}$

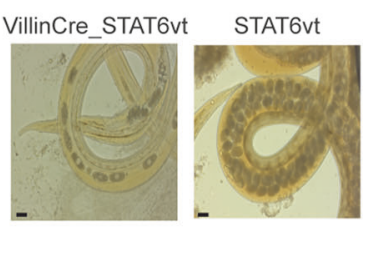

h

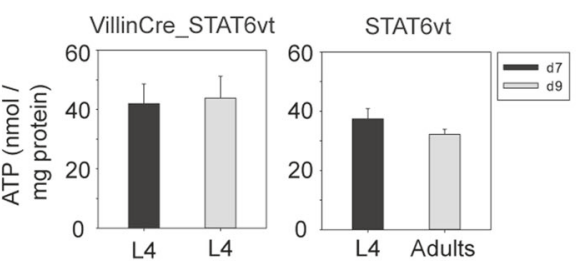

i
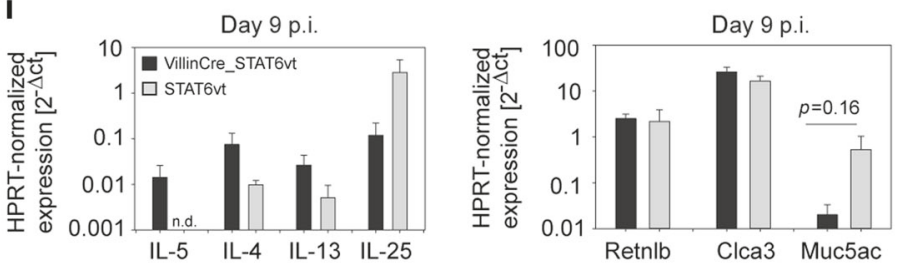

k

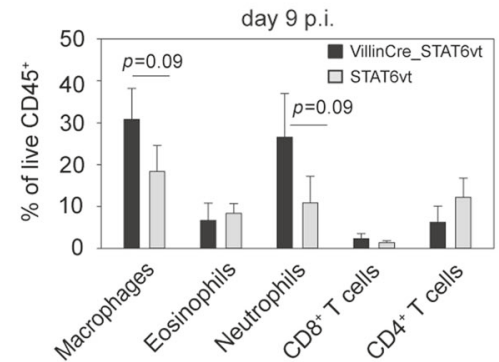

Fig. 6 Two levels of protection by STAT6 signaling in IECs during primary Hp infection. Mice were orally infected with Hp L3. a Representative pictures of the SI with embedded larvae in the submucosa on day 7 or 9 after primary infection (black arrows). b Number of granulomas containing larvae on day 8 after primary infection in VillinCre STAT6vt mice (dark gray bar) and control littermates (light gray bar). c Immunofluorescent staining for Arginase 1 (Arg1, green, upper row), CD68 (green), and Relm- $\alpha$ (red, both middle row) and sialic acid binding Ig-like lectin F (Siglec-F, red, lower row) on frozen sections of larvae-containing granulomas from day 7 after primary infection. Nuclei were counterstained for DAPI (blue). White arrows indicate larvae (Scale bar: $20 \mu \mathrm{m}$ ). d, e Quantitative RT-PCR for M2 macrophage markers (Mrc1, Chil3, Arg1, Pdcd1lg2, and Retnla), the IEC-derived effector protein Relm- $\beta$ (Retnlb), or for collagen synthesis (Col1a1 and LH2) in granulomas on day 8 after primary infection. $\mathbf{f}$ Analysis of parasite burden by quantification of adult worms in the intestinal lumen on day 9 after primary infection and by quantification of eggs per gram feces on days 10, 15, and 20 after primary infection. $g$ Representative pictures of female adult worms isolated out of the SI on day 15 after primary infection (Scale bar: $50 \mu \mathrm{m}$ ). $\mathbf{h}$ Measurement of health of L4 larvae and adults day 7 or 9 after primary infection by quantification of ATP molecules per mg protein content. i Quantitative RT-PCR for cytokines (IL-5, IL-4, IL-13, and IL25) and type 2 immune response associated genes (Retnlb, Clca3, and Muc5ac) day 9 after primary infection. j Immune cells were isolated out of the LP on day 9 after primary $\mathrm{Hp}$ infection and analyzed by FACS. The bar graphs show the percentages of $\mathrm{F} 4 / 80^{+} \mathrm{CD} 11 \mathrm{~b}^{+}$macrophages, Ly6- $\mathrm{C}^{+}$Ly6-G $\mathrm{G}^{+}$neutrophils, $\mathrm{CD} 8^{+}$T cells, and $\mathrm{CD} 4^{+} \mathrm{T}$ cells, all from living $\mathrm{CD} 45^{+}$Siglec- $\mathrm{F}^{-}$cells. In addition, Siglec- $\mathrm{F}^{+}$eosinophils from living CD45 ${ }^{+}$cells are depicted. $\mathbf{k}$ Eggs per gram feces in C57BL/6 mice on days 10, 15, and 21 after primary infection with the indicated amounts of L3. Black graphs in $\mathbf{d}, \mathbf{e}, \mathbf{h}-\mathbf{j}$ represent VillinCre_STAT6vt mice, gray bars represent the negative control littermates. Pictures a, $\mathbf{c}, \mathbf{g}$ are representative of one out of two independent experiments. $\mathbf{b}, \mathbf{d}-\mathbf{f}$ Bars show the mean + s.e.m of pooled data from at least two independent experiments or the mean + s.d. of one experiment $\mathbf{h}-\mathbf{k}$. Two to four mice per group per experiment were used. ${ }^{*} P \leq 0.05,{ }^{* *} P \leq 0.01,{ }^{* * *} P \leq$ 0.001 , unpaired two-tailed Student's $t$ test 
helminth infection. Paneth cells were also increased in VillinCre_STAT6vt mice, and in contrast to characteristic markers for goblet cells and tuft cells, the Paneth cell marker Ang4 was also increased in organoids from these mice. The role of STAT6 in Paneth cells is poorly understood, but IL-13 has been shown to activate release of antimicrobial peptides from Paneth cells. ${ }^{31}$ To what extent Paneth cell-derived factors contribute to protection against helminths remains to be investigated.

We studied protective type 2 immunity in VillinCre_STAT6vt mice using two different helminth infection models because the tissue tropism and developmental program of both pathogens is very different as $\mathrm{Nb}$ causes a transient infection and stays in the lumen while $H p$ enters the submucosa and returns to the lumen after about 8 days to establish a chronic infection. In the $\mathrm{Nb}$ model, we observed efficient worm expulsion even in the absence of CD4 T cells, which are usually required for control of a primary infection in wild-type mice. In fact, worm expulsion was so efficient that only few ILC2s were recruited and much lower levels of IL-13, IL-25, Muc5ac, and Retnlb were observed in the LP of VillinCre_STAT6vt as compared to control mice. However, enough antigens were present to induce a normal Th2 response in the draining $\mathrm{mLNs}$.

The effector molecules and pathways regulated by STAT6controlled genes in IECs are still poorly understood. Relm- $\beta$ is induced in goblet cells in a STAT6-dependent manner and worm expulsion after $\mathrm{Nb}$ and $\mathrm{Hp}$ infection was reported to be severely impaired in Relm- $\beta$-deficient mice. ${ }^{17}$ However, others observed no difference in egg production and expulsion kinetics between $\mathrm{Nb}$ infected WT and Relm- $\beta$-deficient mice. ${ }^{32}$ Other STAT6-regulated effector molecules including mucins, trefoil factors, and intelectins may also contribute to worm expulsion from the intestinal lumen (reviewed in ref. ${ }^{33}$ )

In addition to improved expulsion of adult worms from the lumen of the Sl, we observed efficient trapping of $\mathrm{Hp} \mathrm{L3/L4}$ in the submucosa of VillinCre_STAT6vt mice. Larval trapping correlated with massive accumulation of Arg1-expressing cells around the larval body. This is consistent with a previous study where Arg1expressing M2 macrophages were shown to contribute to protective immunity against secondary $\mathrm{Hp}$ infection by depleting Arginine from the microenvironment of the granulomas and thereby starving the larvae. ${ }^{13}$ Since M2 macrophages were present in granulomas of both strains of mice, we conclude that larval trapping is not caused by more efficient recruitment of M2 macrophages but rather by more efficient expression of Arg1 and the other M2-associated markers within the granulomas. Interestingly, trapped larvae remained alive indicating that they are indeed physically trapped rather than starved due to depletion of Arginine. Exactly how STAT6-induced factors from IECs leads to more efficient expression of $\mathrm{M} 2$-associated effector molecules and collagen during primary $\mathrm{Hp}$ infection in VillinCre_STAT6vt mice remains to be investigated. The few larvae that escaped from the granulomas in VillinCre_STAT6vt mice and entered the lumen on days 8-9 after $H p$ infection were able to develop into adult worms but the females contained only few eggs. This indicates that the fitness of adult $\mathrm{Hp}$ worms is also impaired by STAT6-induced effector molecules from IECs, most likely Relm- $\beta$ based on previous results. $^{17}$

In addition to STAT6-regulated mechanisms of host protection against helminths, there is also evidence for alternative protective pathways. One study has shown that STAT6-independent mechanisms can cause trapping and degradation of adult $\mathrm{Nb}$ in the mucus layer of the SI. ${ }^{34}$ Furthermore, the epithelial cell-derived phospholipase A2 group 1B degrades phospholipids in L3 of $\mathrm{Hp}$ and this enzyme is actually inhibited by IL-4R signaling in organoid cultures. ${ }^{35}$

Taken together, we demonstrate that expression of a constitutively active version of STAT6 in IECs promotes proliferation and differentiation of secretory IECs and orchestrates protective immunity against helminths at two distinct anatomical locations: the submucosa and the lumen of the SI. This finding provides the basis for further investigations on STAT6-regulated genes in IECS that mediate these effects. A better understanding of the mechanisms that control IEC homeostasis and effector functions could help to develop new strategies for treatment of gastrointestinal helminth infections and therapeutic intervention in inflammatory bowel diseases.

\section{METHODS}

Mice

Rosa26 $6^{\text {LSL-STAT6vt }}$ mice were generated by inserting a constitutively active version of STAT6 with a C-terminal FLAG-tag (STAT6vt; ref. ${ }^{20}$ ) behind a loxP-flanked Stop cassette into the genomic Rosa26 locus by standard embryonic stem (ES) cell technology and $\mathrm{pBigT/pROSA26-PA}$ targeting vector system. ${ }^{36} \mathrm{BALB} / \mathrm{c}-\mathrm{I}$ ES cells were used ${ }^{37}$ and homologous recombination was identified by Southern blot analysis of EcoRl-digested genomic DNA. PCR with the following primer pair was used to identify the targeted allele in offspring mice: fwd (5'-CACCATGTCTCTGTGGGGCCTA ATTTCC- $\left.3^{\prime}\right)$ and rev ( $5^{\prime}$-CAGCCCTGCTAGGCTCTCCTCAAAG- $\left.3^{\prime}\right)$. PCR conditions were $5^{\prime} 95^{\circ} \mathrm{C}, 35 \times\left(30^{\prime \prime} 95^{\circ} \mathrm{C}, 30^{\prime \prime} 58^{\circ} \mathrm{C}, 45^{\prime \prime} 72^{\circ} \mathrm{C}\right), 5^{\prime} 72^{\circ}$ $\mathrm{C}$, and the size of the PCR product was $672 \mathrm{bp}$. Rosa26 $6^{\text {LSL-STAT6vt }}$ mice (STAT6vt mice) were then crossed with VillinCre mice (B6.Cg$\mathrm{Tg}$ (Vil1-cre)1000Gum/J, ref. ${ }^{38}$ ) to generate VillinCre_STAT6vt mice. In some experiments, C57BL/6 mice (Charles River, Wilmington, MA) were used.

All mice were housed according to the institutional guidelines and both male and female mice were used at the age of 8-12 weeks. Only healthy mice showing no abnormal behavior were used for the analysis. The animal experiments were approved by the Regierung von Unterfranken and performed in accordance with the German animal protection law.

Helminth infections

Both $\mathrm{Nb}$ and $\mathrm{Hp}$ were used as worm infection models. Five hundred $500 \mathrm{Nb}$ L3 were injected subcutaneously at the base of the tail. For the first 5 days after infection, the mice were given antibiotics-containing drinking water $(2 \mathrm{~g} / \mathrm{l}$ neomycin sulfate, 100 mg/l polymyxin B; Sigma-Aldrich, St. Louis, MO). For Hp infection, mice were orally gavaged with 200 L3. For determination of parasite egg burden, fecal pellets were moistened overnight (o.n.) in $1 \mathrm{ml}$ water and then counted in a McMaster counting chamber (FiBL, Frick, Switzerland) after adding $3 \mathrm{ml}$ of saturated sodium chloride (Merck Millipore, Darmstadt, Germany). For analysis of adult worm burden, the SI was cut open longitudinally and incubated for $2 \mathrm{~h}$ at $37^{\circ} \mathrm{C}$ in RPMI-1640 medium (PanBiotech, Aidenbach, Germany).

RNA extraction, cDNA synthesis, and quantitative RT-PCR RNA was isolated out of tissue pieces or small intestinal organoids according to the instructions of the RNeasy Mini Kit from Qiagen (Qiagen, Hilden, Germany). In all, $2 \mu \mathrm{g}$ of RNA were used for reverse transcription to CDNA (High Capacity Reverse Transcription Kit, ThermoFisher, Waltham, MA) followed by quantitative RT-PCR using the SYBR-Green method (SYBR Select Master Mix, ThermoFisher). The following primer pairs were used: Porphobilinogen deaminase (PBGD) forward 5'-GTGGAAGTCC GAGCCAAGGA-3', PBGD reverse 5'-CTAGCTGGGCTCCTCTTGGA-3', Hypoxanthine-guanine phosphoribosyltransferase 1 (HPRT1) forward 5'-GTTGGATACAGGCCAGACTITGTT-3', HPRT1 reverse 5'GAGGGTAGGCTGGCCTATAGGCT-3', Retnlb forward 5'-AAGCCTA CACTGTGTITCCTIT-3', Retnlb reverse 5'-GCTTCCTTGATCCTाT GATCCAC-3', Ang4 forward 5'-CTGTCTCCAGGAGCACACAGCTA G-3', Ang4 reverse 5'-GCTTGGCATCATAGTGCTGACG-3', Chil3 forward 5'-GGAATTGGTGCCCCTACAAT-3', Chil3 reverse 5'-CCAG ACCTCAGTGGCTCCT-3', Retnla forward 5'-CCATAGAGAGATTATC 
GTGGA-3', Retnla reverse 5'-TGGTCGAGTCAACGAGTAAG-3', Arg1 forward 5'-GTATGACGTGAGAGACCACG-3', Arg1 reverse 5'CTCGCAAGCCAATGTACACG-3', Pdcd1lg2 forward 5'-CTGCCGATA CTGAACCTGAGC-3', Pdcd1lg2 reverse 5'-GCGGTCAAAATCGCAC TCC-3', Lgr-5 forward 5'-ACCCGCCAGTCTCCTACATC-3', Lgr-5 reverse 5'-GCATCTAGGCGCAGGGATTG-3', IL-25 forward 5'-ACCAC AACCAGACGGTCTTC-3', IL-25 reverse 5'-CTGCTTCAGGTAGG GCTITG-3', Mrc1 forward 5'-GCAAACATTGGGCAGAAGG-3', Mrc1 reverse 5'-AGGAAACGGGAGAACCATC-3', doublecortin-like kinase 1 chloride channel accessory 1 (DCLK1) forward 5'-CAAGCCAGCC ATGTCGTTC-3', DCLK1 reverse 5'-TTCCTITGAAGTAGCGGTCAC-3', (Clca3) forward 5'-GCAGTGAGGTGTTCAGCAGC-3', Clca3 reverse 5'GGTCATTTGGGGCTTCTTGATTGTG-3', IL-4 forward 5'-ACTTGAGAG AGATCATCGGCA-3', IL-4 reverse 5'-AGCTCCATGAGAACACTAGA GTT-3', IL-5 forward 5'-TCTGTACTCATCACACCAAG-3', IL-5 reverse 5'-AGGATGCTTCTGCACTTGAG-3', IL-13 forward 5'-TGCTTGT GTAGCTGAGCAG -3', IL-13 reverse 5'-GCAGTCCTGGCTCTTGCTTG3', Muc5ac forward 5'-CTGTGACATTATCCCATAAGCCC-3', Muc5ac reverse $5^{\prime}$-AAGGGGTATAGCTGGCCTGA, Math1 forward 5'-GTGGA CGAGCTTGGCTGC-3', Math1 reverse $5^{\prime}$-CTTGTCGTTGTTGAAGGA CGGG-3', Hes1 forward 5'-GGAGAAGAGGCGAAGGGCAAG-3', Hes1 reverse 5'-CCGGAGGTGCTTCACAGTCATTTC-3', Sox-9 forward 5'-G ACCCTTCGTGGAGGAGGC-3', Sox-9 reverse 5'-CGCTTCAGATCAA CTITGCCAGC-3', Sox-4 forward 5'-GGCGGCTGCATCGTTCTC-3', Sox-4 reverse 5'-CGCGCTTCACTITCTTGTCGG-3', SPDEF forward 5'-GATATTGAGACGGCCTGCAAGCTTC-3', SPDEF reverse 5'-CTT CCAGATGTCCAGGTGGGC-3', LH2 forward 5'-CGCCAGTCAGGAAGA TCTGGTC-3', LH2 reverse 5'-CTCCAGAATTCAGGTAGCGTTTCCC-3', Dll4, villin 1 (Villin), and Pofut1 primer were kindly provided by $C$. Günther (all QIAGEN Quantitect primer assays). PBGD and HPRT1 served as housekeeping genes (All primers were purchased from Microsynth AG, Balgach, Switzerland). The PCR cycling conditions were as follows: $95^{\circ} \mathrm{C}, 5 \mathrm{~min} ; 95^{\circ} \mathrm{C}, 30 \mathrm{~s} ; 58^{\circ} \mathrm{C}, 30 \mathrm{~s}$; $72{ }^{\circ} \mathrm{C}, 45 \mathrm{~s}$ (39 cycles).

Western blot

Tissue pieces were snap frozen and homogenized in the appropriate volume of RIPA lysis buffer (1\% NP-40, $50 \mathrm{mM}$ Tris $\mathrm{pH} 7.4,0,15 \mathrm{M} \mathrm{NaCl}, 1 \mathrm{mM}$ EDTA pH 8.0, 0,25\% deoxycholic acid) using a TissueLyzer (Qiagen). Organoids were lysed directly in the well using the RIPA lysis buffer. The samples were incubated on ice for $30 \mathrm{~min}$ and centrifuged at $21912 \times g$ for $20 \mathrm{~min}$. The supernatant was transferred in a new tube and the protein concentration was determined by BCA assay (ThermoFisher). The samples were run on a Mini/Midi TGX precast protein gel and blotted on a polyvinylidene difluoride mini/midi membrane using the Trans-Blot Turbo Transfer System (BioRad, Munich, Germany). After blocking of the membrane, the primary antibody was incubated o.n. at $4{ }^{\circ} \mathrm{C}$ (STAT6-M20, Santa Cruz, Dallas, TX; pSTAT6 Tyr641, Cell Signaling Technology, Danvers, MA; Relm- $\beta$, PeproTech, Rocky Hill, NJ). Horseradish peroxidase goat anti-rabbit-lgG was used as secondary antibody, followed by adding the substrate Signal Fire ${ }^{T M}$ Elite ECL Reagent (Cell Signaling Technology). The signal was detected using the ChemiDoc ${ }^{\mathrm{TM}}$ Imaging System (BioRad). For determination of Relm- $\beta$ protein in the feces, one fecal pellet per mouse was homogenized in $500 \mu \mathrm{l}$ of RIPA lysis buffer.

\section{Flow cytometry}

LP lymphocytes were isolated out of the SI of naive or infected mice as described elsewhere. ${ }^{15} \mathrm{mLN}$ cells were smashed through a $70-\mu \mathrm{m}$ cell strainer (Corning, UK) and centrifuged for $5 \mathrm{~min}, 400 \times$ $g$ at $4{ }^{\circ} \mathrm{C}$.

Cells were blocked with antiCD16/CD32 antibody (2.4G2, BioXcell, West Lebanon, NH) in phosphate-buffered saline (PBS, Merck Millipore, Darmstadt, Germany) containing 2\% fetal bovine serum (Gibco, ThermoFisher) prior to surface or intracellular staining. Antibodies were incubated at $4{ }^{\circ} \mathrm{C}$ for $20 \mathrm{~min}$. For intracellular staining of cytokines, the cells were fixed with $4 \%$ para-formaldehyde (PFA, Carl Roth, Karlsruhe, Germany) at room temperature for $20 \mathrm{~min}$, followed by adding permeabilization buffer (BioLegend, San Diego, USA). The antibodies were purchased from Miltenyi (Miltenyi Biotec, Bergisch Gladbach, Germany), eBioscience (ThermoFisher), BD Bioscience, or Biolegend and conjugated to biotin, APC-Cy7, fluorescein isothiocyanate, AF488, allophycocyanin (APC), AF405, Amcyan, e450, PacificBlue, Brilliant-violet BV421, phycoerythrin (PE)-Cy7, $\mathrm{PE}$, or PerCp-Cy5.5. The following antibodies were used: CD3e (clone 145-2C11), CD8e (53-6.7), CD11b (M1/70), CD49b (HMa2), B220 (RA3-6B2), Gr-1 (RB6-8C5), Ter119 (TER-119), Tcry (eBioGL3), NK1.1 (PK136), CD90.1/2 (30-H12/HIS51), Ly5.1/2 (A20/104), Sca-1 (D7), CD4 (RM4-5), KLRG1 (2F1), CD4 (RM 4-5), CD44 (IM7.8.1), CD62L (MEL 14), IL4 (11B11), IL13 (eBio13A), Siglec-F (E50-2440), Ly6-C (HK1.4), Ly6-G (1A8), CD200R3 (Ba13), and F4/80 (BM8). For differentiation between living and dead cells, Fixable Viability Dye eFluor $^{\mathrm{TM}} 780$ was used. The cells were acquired on a BD FACSCanto $^{\text {TM }}$ II or BD LSRFortessa ${ }^{T M}$ (BD Bioscience, San Jose, CA).

Phorbol-12-myristate-13-acetate (PMA)/lonomycin restimulation Cell suspensions of $\mathrm{mLNs}$ were restimulated using $2 \mu \mathrm{g} / \mathrm{ml}$ lonomycin and $40 \mathrm{ng} / \mathrm{ml}$ PMA (both Sigma-Aldrich). After $2 \mathrm{~h}$, medium containing $50 \mu \mathrm{g} / \mathrm{ml}$ Brefeldin A (Sigma-Aldrich) was added to the cells. Cells were harvested after $5 \mathrm{~h}$ and analyzed by flow cytometry.

\section{BCA assay and ELISA}

Protein concentration was determined by using the Pierce ${ }^{T M}$ BCAProtein Assay Kit (ThermoFisher). Serum IgE levels of naive and $\mathrm{Nb}$ infected mice as well as IL-4 and IL-13 levels in supernatants of $\mathrm{PMA} /$ lonomycin restimulated cells were determined by ELISA. Anti-lgE (R35-72, BD Pharmingen) or anti-IL-4 (11B11, BioLegend) was used for coating and biotinylated anti-lgE (R35-118, BD Pharmingen) or biotinylated anti-IL-4 (BVD6-24G2, BioLegend) was used for detection. IL-13 levels were measured with the ELISA Kit from PeproTech.

\section{Generation of small intestinal organoids}

Small intestinal organoids were generated as previously described. ${ }^{39}$ After 4 days of culture, organoids were washed, resuspended in fresh matrigel, and transferred into new wells. Organoids were harvested on day 7 of culture for isolation of RNA or on day 10 for western blot analysis.

Histology

One-cm cylinders of the respective area of the SI of naive or infected mice were isolated and flushed with PBS. Samples were fixed in $4 \%$ PFA o.n. at $4{ }^{\circ} \mathrm{C}$ and embedded in Paraffin (Engelbrecht, Edermünde, Germany) or tissue-freezing medium for cryosectioning (Leica, Wetzlar, Germany). Paraffin-embedded samples were dewaxed at $60^{\circ} \mathrm{C}$ for $20 \mathrm{~min}$, immersed two times in xylol (Merck Millipore), and hydrated to water using decreasing ethanol concentrations (Carl Roth). Frozen sections were shortly thawed at $42{ }^{\circ} \mathrm{C}$ and rehydrated in PBS. For retrieval of antigens, samples were heated in a pressure cooker containing citrate buffer for $10 \mathrm{~min}$ (10 mM sodium citrate, $0.05 \%$ Tween $20, \mathrm{pH} 6.0$ ). Samples were blocked with TNB buffer (Perkin Elmer, Waltham, MA) containing $40 \mu \mathrm{g} / \mathrm{ml}$ antiCD16/CD32 antibody, 2\% mouse serum, and $2 \%$ serum of the host the secondary antibody was raised in. Sections were stained for neutral mucopolysaccharides using the periodic acid Schiff reaction (Merck Millipore), tuft cells (Rabbit anti-mouse DCLK1, abcam, Cambridge, UK), Paneth cells (Rabbit anti-mouse Lysozyme, ThermoFisher), Relm-a (Rabbit antimouse, abcam), CD68-A488 (FA-11, Biolegend), rat-anti-mouseSiglec-F (E50-2440, BD) or Arginase-1 (V-20, Santa Cruz). Cy ${ }^{\mathrm{TM} 3}$ goat anti-rabbit IgG or donkey anti-rabbit lgG-A647 (both Jackson ImmunoResearch Laboratories, West Grove, PA), and goat anti-rat 
IgG-A488 (Poly4054, Biolegend) were used as secondary antibodies. Cell nuclei were counterstained with 4,6 diamidino-2phenylindole (DAPI) or Mayer's Hematoxylin solution (SigmaAldrich).

Immunofluorescence staining of BrdU

BrdU (1 mg; Sigma-Aldrich) per mouse was injected i.p. After $24 \mathrm{~h}$, pieces of the ileum were isolated, fixed o.n. in 4\% PFA, and embedded in tissue-freezing medium for cryosectioning. The slides were treated with $1 \mathrm{M} \mathrm{HCl}$ (Carl Roth) for $10 \mathrm{~min}$ on ice, followed by $2 \mathrm{M} \mathrm{HCl}$ treatment for $10 \mathrm{~min}$ at room temperature. Sections were blocked with $40 \mathrm{\mu g} / \mathrm{ml}$ antiCD16/CD32 antibody and $2 \%$ mouse serum. In addition, binding sites of endogenous biotin were blocked using the Streptavidin/Biotin Blocking Kit (Vector Laboratories, Burlingame, CA). Incorporated BrdU was detected with biotinylated anti-BrdU (Bu20a, ThermoFisher) o.n. at $4{ }^{\circ} \mathrm{C}$ followed by Streptavidin-Cy3 incubation (Jackson ImmunoResearch Laboratories). Cell nuclei were counterstained with DAPI.

\section{Cytospin}

Single-cell suspensions of IECs were generated by using the first steps of the LP Dissociation Kit (Miltenyi). Cells were spinned down on a coverslip using a cytospin apparatus (Shannon, ThermoFisher). Cells were immediately fixed with ice-cold methanol (Carl Roth) blocked and stained for either pSTAT6 or fluorophoreconjugated EpCAM (caa7-9G8, Miltenyi Biotec). Cy ${ }^{\text {TM} 3}$ goat antirabbit IgG (Jackson ImmunoResearch Laboratories) was used as the secondary antibody.

\section{ATP assay}

For analysis of larval or adult health of $\mathrm{Hp}$ parasites, CellTiter-Glo ${ }^{\circledR}$ Luminescent Cell Viability Assay was used (Promega, Madison, WI). Larvae or adults were isolated out of the submucosa or the lumen of the SI, washed extensively, and homogenized using a Bead Ruptor (Omni, Kennesaw, GA). Luminescence was recorded using a Luminoskan Ascent device (ThermoFisher). The ATP concentration in nanomoles per mg protein was analyzed.

\section{Microscopy}

Images were taken on a confocal microscope LSM700 or Axio VERT.A1 and analyzed using the ZEN software (All Carl Zeiss, Jena, Germany).

\section{Statistics}

The sample size was chosen according to preliminary observations. The data were analyzed using SigmaPlot (12.3, Systat Software, San Jose, CA). All data are represented as means + standard deviation or standard error. Data were tested for statistical significance by using an unpaired two-tailed Student's $t$ test. Levels of significance: ${ }^{*} P \leq 0.05,{ }^{* *} P \leq 0.01,{ }^{* *} P \leq 0.001$.

\section{ACKNOWLEDGEMENTS}

We thank K. Castiglione, D. Döhler, and L. Handl for technical assistance; M. Kirsch and C. Dietz for animal husbandry; H. Nakajima for reagents; T. Winkler and D. Engelkamp for ES cell injection; R. Palmisano and T. Fraass from the Opical Imaging Center Erlangen (OICE) for help with fluorescence microscopy; and members of the Voehringer laboratory for helpful discussions. This work was supported by the Deutsche Forschungsgemeinschaft (DFG) with grants CRC1181_A02, TRR130_TP20, and VO 944/8-1 to D.V. and grants CRC1181_C05, TRR241_A03, and KFO257 to C.B.

\section{AUTHOR CONTRIBUTIONS}

C.K., B.K., and D.V. conceived the study; C.K., B.K., M.O., and C.S. performed experiments; E.M., C.G., and C.B. provided mice and expertise for organoid cultures and histology; C.D. provided instrumentation for histology; C.K. analyzed data and generated figures; C.K. and D.V. wrote the manuscript.

\section{ADDITIONAL INFORMATION}

The online version of this article (https://doi.org/10.1038/s41385-018-0107-3) contains supplementary material, which is available to authorized users.

Competing interests: The authors declare no competing interests.

Data availability: All data are available from the authors upon request.

\section{REFERENCES}

1. Hotez, P. J. et al. Helminth infections: the great neglected tropical diseases. J. Clin. Investig. 118, 1311-1321 (2008)

2. Hotez, P. J., Bethony, J. M., Diemert, D. J., Pearson, M. \& Loukas, A. Developing vaccines to combat hookworm infection and intestinal schistosomiasis. Nat. Rev. Microbiol. 8, 814-826 (2010).

3. Kim, D. G. et al. Intestinal nematodes from small mammals captured near the demilitarized zone, Gyeonggi province, Republic of Korea. Korean J. Parasitol. 53, 135-139 (2015).

4. Katona, I. M., Urban, J. F. Jr. \& Finkelman, F. D. The role of L3T4+ and Lyt-2+ $\mathrm{T}$ cells in the $\mathrm{IgE}$ response and immunity to Nippostrongy/us brasiliensis. J. Immunol. 140, 3206-3211 (1988).

5. Neill, D. R. et al. Nuocytes represent a new innate effector leukocyte that mediates type-2 immunity. Nature 464, 1367-1370 (2010).

6. Oeser, K., Schwartz, C. \& Voehringer, D. Conditional IL-4/IL-13-deficient mice reveal a critical role of innate immune cells for protective immunity against gastrointestinal helminths. Mucosal Immunol. 8, 672-682 (2015).

7. Voehringer, D., Reese, T. A., Huang, X., Shinkai, K. \& Locksley, R. M. Type 2 immunity is controlled by IL-4/IL-13 expression in hematopoietic non-eosinophil cells of the innate immune system. J. Exp. Med. 203, 1435-1446 (2006).

8. Howitt, M. R. et al. Tuft cells, taste-chemosensory cells, orchestrate parasite type 2 immunity in the gut. Science 351, 1329-1333 (2016).

9. Gerbe, F. et al. Intestinal epithelial tuft cells initiate type 2 mucosal immunity to helminth parasites. Nature 529, 226-230 (2016).

10. von Moltke, J., Ji, M., Liang, H. E. \& Locksley, R. M. Tuft-cell-derived IL-25 regulates an intestinal ILC2-epithelial response circuit. Nature 529, 221-225 (2016).

11. Gause, W. C., Urban, J. F. Jr. \& Stadecker, M. J. The immune response to parasitic helminths: insights from murine models. Trends Immunol. 24, 269-277 (2003).

12. Morimoto, M. et al. Peripheral CD4 T cells rapidly accumulate at the host: parasite interface during an inflammatory Th2 memory response. J. Immunol. 172, 2424-2430 (2004).

13. Anthony, R. M. et al. Memory $\mathrm{T}(\mathrm{H}) 2$ cells induce alternatively activated macrophages to mediate protection against nematode parasites. Nat. Med. 12, 955-960 (2006).

14. McCoy, K. D. et al. Polyclonal and specific antibodies mediate protective immunity against enteric helminth infection. Cell Host Microbe 4, 362-373 (2008).

15. Schwartz, C. et al. Basophil-mediated protection against gastrointestinal helminths requires IgE-induced cytokine secretion. Proc. Natl Acad. Sci. USA 111, E5169-E5177 (2014).

16. Nelms, K., Keegan, A. D., Zamorano, J., Ryan, J. J. \& Paul, W. E. The IL-4 receptor: signaling mechanisms and biologic functions. Annu. Rev. Immunol. 17, 701-738 (1999).

17. Herbert, D. R. et al. Intestinal epithelial cell secretion of RELM-beta protects against gastrointestinal worm infection. J. Exp. Med. 206, 2947-2957 (2009).

18. Horsnell, W. G. et al. Delayed goblet cell hyperplasia, acetylcholine receptor expression, and worm expulsion in SMC-specific IL-4Ralpha-deficient mice. PLoS Pathog. 3, e1 (2007).

19. Daniel, C., Salvekar, A. \& Schindler, U. A gain-of-function mutation in STAT6. J. Biol. Chem. 275, 14255-14259 (2000).

20. Mikita, T., Daniel, C., Wu, P. \& Schindler, U. Mutational analysis of the STAT6 SH2 domain. J. Biol. Chem. 273, 17634-17642 (1998).

21. Crosnier, C., Stamataki, D. \& Lewis, J. Organizing cell renewal in the intestine: stem cells, signals and combinatorial control. Nat. Rev. Genet. 7, 349-359 (2006).

22. Mori-Akiyama, Y. et al. SOX9 is required for the differentiation of paneth cells in the intestinal epithelium. Gastroenterology 133, 539-546 (2007).

23. Gracz, A. D. et al. SOX4 promotes ATOH1-independent intestinal secretory differentiation toward tuft and enteroendocrine fates. Gastroenterology 155, 1508. e10-1523.e10 (2018).

24. Knipper, J. A. et al. Interleukin-4 receptor alpha signaling in myeloid cells controls collagen fibril assembly in skin repair. Immunity 43, 803-816 (2015).

25. Wills-Karp, M. \& Finkelman, F. D. Untangling the complex web of IL-4- and IL-13mediated signaling pathways. Sci. Signal. 1, pe55 (2008).

26. Urban, J. F. Jr. et al. IL-13, IL-4Ralpha, and Stat6 are required for the expulsion of the gastrointestinal nematode parasite Nippostrongylus brasiliensis. Immunity $\mathbf{8}$, 255-264 (1998). 
424

27. Finkelman, F. D. et al. Interleukin-4- and interleukin-13-mediated host protection against intestinal nematode parasites. Immunol. Rev. 201, 139-155 (2004).

28. Khan, W. I., Blennerhasset, P., Ma, C., Matthaei, K. I. \& Collins, S. M. Stat6 dependent goblet cell hyperplasia during intestinal nematode infection. Parasite Immunol. 23, 39-42 (2001).

29. Voehringer, D., Shinkai, K. \& Locksley, R. M. Type 2 immunity reflects orchestrated recruitment of cells committed to IL-4 production. Immunity 20, 267-277 (2004).

30. Cliffe, L. J. et al. Accelerated intestinal epithelial cell turnover: a new mechanism of parasite expulsion. Science 308, 1463-1465 (2005).

31. Stockinger, S. et al. Interleukin-13-mediated paneth cell degranulation and antimicrobial peptide release. J. Innate Immun. 6, 530-541 (2014).

32. Chen, G., Wang, S. H., Jang, J. C., Odegaard, J. I. \& Nair, M. G. Comparison of RELMalpha and RELMbeta single- and double-gene-deficient mice reveals that relmalpha expression dictates inflammation and worm expulsion in hookworm infection. Infect. Immun. 84, 1100-1111 (2016).
33. Sharpe, C., Thornton, D. J. \& Grencis, R. K. A sticky end for gastrointestinal helminths; the role of the mucus barrier. Parasite Immunol. 40, e12517 (2018).

34. Van Panhuys, N. et al. Mucosal trapping and degradation of Nippostrongylus brasiliensis occurs in the absence of STAT6. Parasitology 140, 833-843 (2013).

35. Entwistle, L. J. et al. Epithelial-cell-derived phospholipase A2 group $1 B$ is an endogenous anthelmintic. Cell Host Microbe 22, 484-493 (2017). e485.

36. Srinivas, S. et al. Cre reporter strains produced by targeted insertion of EYFP and ECFP into the ROSA26 locus. BMC Dev. Biol. 1, 4 (2001).

37. Noben-Trauth, N., Kohler, G., Burki, K. \& Ledermann, B. Efficient targeting of the IL4 gene in a BALB/C embryonic stem cell line. Transgenic Res. 5, 487-491 (1996).

38. Madison, B. B. et al. Cis elements of the villin gene control expression in restricted domains of the vertical (crypt) and horizontal (duodenum, cecum) axes of the intestine. J. Biol. Chem. 277, 33275-33283 (2002).

39. Sato, T. \& Clevers, H. Primary mouse small intestinal epithelial cell cultures. Methods Mol. Biol. 945, 319-328 (2013). 